\title{
Associations between potentially functional CORIN SNPs and serum corin levels in the Chinese Han population
}

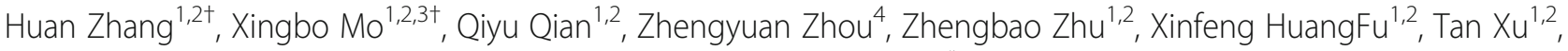
Aili Wang ${ }^{1,2}$, Zhirong Guo ${ }^{1,2}$, Shufeng Lei ${ }^{1,2,3}$ and Yonghong Zhang ${ }^{1,2^{*}}$

\begin{abstract}
Background: Corin is an important convertase involved in the natriuretic peptide system and may indirectly regulate blood pressure. Genetic factors relate to corin remain unclear. The purpose of the current study was to comprehensively examine the associations among CORIN SNPs, methylations, serum corin levels and hypertension.

Results: We genotyped 9 tag-SNPs in the CORIN gene and measured serum corin levels in 731 new-onset hypertensive cases and 731 age- and sex-matched controls. DNA methylations were tested in 43 individuals. Mendelian randomization was used to investigate the causal associations. Under additive models, we observed associations of rs 2289433 (p.Cys13Tyr), rs6823184, rs10517195, rs2271037 and rs12509275 with serum corin levels after adjustment for covariates ( $P=0.0399,0.0238,0.0016,0.0148$ and 0.0038 , respectively). The tag-SNP rs6823184 and SNPs that are in strong linkage disequilibrium with it, i.e., rs10049713, rs6823698 and rs1866689, were associated with CORIN gene expression $\left(P=2.38 \times 10^{-24}, 5.94 \times 10^{-27}, 6.31 \times 10^{-27}\right.$ and $6.30 \times 10^{-27}$, respectively). Neither SNPs nor corin levels was found to be associated with hypertension. SNP rs6823184, which is located in a DNase hypersensitivity cluster, a CpG island and transcription factor binding sites, was significantly associated with cg02955940 methylation levels $\left(P=1.54 \times 10^{-7}\right)$. A putative causal association between cg02955940 methylation and corin levels was detected $(P=0.0011)$.
\end{abstract}

Conclusion: This study identified potentially functional CORIN SNPs that were associated with serum corin level in the Chinese Han population. The effect of CORIN SNPs on corin level may be mediated by DNA methylation.

Keywords: Corin, Natriuretic peptide, Hypertension, Methylation, Mendelian randomization

\section{Background}

Hypertension is identified as one of the most important causal risk factors for cardiovascular diseases, which is the leading cause of death worldwide $[1,2]$. The levels of diagnosis, treatment and control of hypertension are much lower in China than in Western countries. The China Kadoorie Biobank Study, a nationwide investigation in 500,223 people, showed that the prevalence of

\footnotetext{
* Correspondence: yhzhang@suda.edu.cn

${ }^{+}$Huan Zhang and Xingbo Mo contributed equally to this work.

'Jiangsu Key Laboratory of Preventive and Translational Medicine for

Geriatric Diseases, Medical College of Soochow University, Suzhou, Jiangsu,

People's Republic of China

${ }^{2}$ Department of Epidemiology, School of Public Health, Medical College of Soochow University, Suzhou, 199 Renai Road, Industrial Park District, Suzhou 215123, Jiangsu, People's Republic of China

Full list of author information is available at the end of the article
}

hypertension has already increased to $32.5 \%$ during year 2004 to 2009 [3].

The natriuretic peptide system regulates salt, water balance and blood pressure (BP) [4]. Corin, a type II transmembrane serine protease found in cardiomyocytes, converts the precursor molecules of A-type natriuretic peptides (ANP) into active proteins [5]. Deficiency in corin is expected to reduce ANP production and cause hypertension [5]. Variants in the human CORIN gene have been identified to alter corin protein conformation and inhibited corin zymogen activation and contribute to hypertension and cardiovascular diseases [6, 7].

The prospective association of serum corin level with hypertension has not been assessed in a Chinese Han population. On the other hand, genetic and epigenetic

(c) The Author(s). 2019 Open Access This article is distributed under the terms of the Creative Commons Attribution 4.0 International License (http://creativecommons.org/licenses/by/4.0/), which permits unrestricted use, distribution, and reproduction in any medium, provided you give appropriate credit to the original author(s) and the source, provide a link to the Creative Commons license, and indicate if changes were made. The Creative Commons Public Domain Dedication waiver (http://creativecommons.org/publicdomain/zero/1.0/) applies to the data made available in this article, unless otherwise stated. 
factors that affect serum corin level have not been identified. What's more, although previous genetic association studies examined the associations of CORIN SNPs with hypertension $[8,9]$, few studies have tried to explore the functional variants to explain the associations and mechanisms [10]. Therefore, we conducted this study to (1) evaluate the effect of serum corin level on hypertension, (2) evaluate the effects of SNPs in CORIN gene on serum corin level and (3) hypertension, and (4) find out the potentially functional variants. Firstly, we examined the association between serum corin level and new-onset hypertension in a nested case-control study from a large prospective cohort of non-hypertensive people who were followed-up over a 6-year period. Then we tested whether SNPs in CORIN gene were associated with baseline serum corin level and hypertension in the same sample. In addition, we evaluated the functionalities of the associated SNPs and SNPs in strong linkage disequilibrium (LD) with them by bioinformatics analysis. Finally, we applied Mendelian randomization to identify putative causal factors, e.g., DNA methylation.

\section{Methods}

\section{Study population}

The study was a nested case-control study from a large prospective cohort of non-hypertensive people in Jiangsu Province, China. The baseline survey among 12,423 participants has been completed in 2007-2008. Participants with cardiovascular diseases, diabetes mellitus, nephropathy, hypertension, or were taking antihypertensive medication were excluded at baseline. The follow-up study has been completed in 2013, and a total of 1774 participants were found to be hypertensive [11]. Among the 1774 patients with new-onset hypertension, we selected 740 new-onset hypertension patients who have both serum and DNA samples as cases by means of stratified random sampling method according to their resident areas. We selected half of the 1774 incident hypertensive individuals because the high cost of the testing of serum corin levels and genotyping. The paired controls were chosen in the follow-up study. Written informed consent was obtained from each of the study participants. This study was approved by the ethics committee at Soochow University in China.

\section{Baseline examination}

The survey methods have been described previously [12]. In brief, basic information for all participants was obtained using a standard questionnaire administered by trained staff. Three sitting BP measurements were taken at $30-\mathrm{s}$ intervals by trained observers using a standard mercury sphygmomanometer after the subjects had been resting for $5 \mathrm{~min}$ according to a standard protocol. The first and fifth Korotkoff sounds were recorded as the systolic (SBP) and diastolic (DBP) blood pressures, respectively. The mean of the three BP measurements was used in the analysis. Fasting blood samples were collected for laboratory tests, including total cholesterol (TC), triglycerides (TG), high-density lipoprotein cholesterol (HDL-C), and fasting blood glucose (FBG). Low-density lipoprotein cholesterol (LDL-C) levels were calculated using the Friedewald equation for participants who had less than $400 \mathrm{mg} / \mathrm{dL}$ TG.

\section{Follow-up study}

All participants were followed up in 2013. The information of demographic characteristics, lifestyle risk factors, personal medical history and family history of hypertension for all participants was also obtained using a standard questionnaire administered by trained staff. The questionnaires and measuring methods used in the follow-up study were the same as those used at baseline. Three sitting BP measurements were taken at 30-s intervals by trained observers using a standard mercury sphygmomanometer after the subjects had been resting for $5 \mathrm{~min}$ according to a standard protocol. The first and fifth Korotkoff sounds were recorded as the SBP and DBP, respectively. The mean of the three $\mathrm{BP}$ measurements was used in the analysis. Hypertension was defined as $\mathrm{SBP} \geq 140 \mathrm{mmHg}$ and/or $\mathrm{DBP} \geq 90 \mathrm{mmHg}$ or use of antihypertensive medication in the last 2 weeks [13]. Sex- and age-matched ( \pm 2 years) controls were selected. The controls were normotensive participants during the follow-up throughout the same time interval as the hypertensive cases.

\section{Measurement of corin}

Blood samples were collected for all of the participants in the morning after at least $8 \mathrm{~h}$ of fasting. The serum samples were frozen at $-80^{\circ} \mathrm{C}$ until the laboratory testing was performed. We used ELISA kits (R\&D Systems, Minneapolis, MN; Catalog: DCRN00) to test the serum corin levels. All analyses were performed by the same lab and all of the samples were processed in a duplicate assay. Standard curves were constructed from which corin concentrations of unknown samples were determined. Intra- and interassay coefficients of variation were less than $5 \%$.

\section{SNP selection and genotyping}

We selected tag-SNPs for the CORIN gene by using the Haploview software (version 4.2, http://www. broad.mit.edu/mpg/haploview) with $\mathrm{LD} \mathrm{r}^{2}$ thresholds of 0.8 [14]. A total of 9 tag-SNPs were selected and genotyped by using SNPscan ${ }^{\text {Tu }}$ technology, a custom- 
by-design 48-Plex Kit based on double ligation and multiplex fluorescence PCR (Catalog: G0104; Genesky Biotechnologies Inc., Shanghai, China). This kit was developed according to patented SNP genotyping technology by Genesky Biotechnologies Inc., which was based on double ligation and multiplex fluorescence PCR.

\section{Detection of potentially functional variants}

We detected putative functional variants which are in strong LD $\left(r^{2}>0.8\right.$, based on data of 1000G Phase 1 of East Asian population) with the tag-SNPs that were detected to be associated with serum corin level or hypertension. Several kinds of functional variants were considered in this study, including missense mutations, SNPs with specific functions such as $\mathrm{N}^{6}$ methyladenosine $\left(\mathrm{m}^{6} \mathrm{~A}\right)$-associated SNPs [15] and phosphorylation-related SNPs (phosSNPs) [16], SNPs have regulatory potentials, as well as expression quantitative trait loci (eQTL) in CORIN. The $\mathrm{m}^{6} \mathrm{~A}$-associated SNPs were found in the m6AVar database (http://m6avar.renlab.org/), and the phosSNPs were found in the phosSNP 1.0 database (http://phossnp. biocuckoo.org/index.php). eQTLs and SNPs with potential functionalities in transcription regulation were found in HaploReg (https://pubs.broadinstitute.org/ mammals/haploreg/haploreg.php).

\section{Causal inference}

We examined whether the identified SNPs were associated with methylation levels by using data extracted from our in-house datasets of 43 female subjects from the Chinese Han population (GSE111942). Basic characteristics of the study subjects have been described in a previous study [17]. The study was approved by the ethical committee of Soochow University. The written informed consent was obtained from each of the participants. Affymetrix Genome-Wide Human SNP Array 6.0 chips were employed for SNP genotyping. DNA methylation profiling was performed using Illumina $450 \mathrm{~K}$ Infinium Methylation BeadChip (Illumina, Inc., USA) according to the manufacturer's instructions. The associations between SNPs and methylations were detected by using the $\mathrm{R}$ package MatrixeQTL [18]. Finally, we applied the gsmr R-package which implements the Generalised Summary-data-based Mendelian Randomization (GSMR) method $[15,19]$ to test for putative causal association between methylation and corin levels using summary-level data on the associations of SNPs with methylations and associations of the same SNPs with corin levels. Compare with other Mendelian randomization approaches, the GSMR method doesn't need individual-level raw data that was tested in the same samples, and it can work without data on the associations between methylation levels (risk factor) and corin levels (outcome). This method facilitates the integration of the methylation data and the corin level data from independent studies via the instrument variables. The tested SNPs that were significantly associated with methylation levels (Bonferroni correction $P<5.56 \times 10^{-3}$ ) were used as instrument variables, because a strong association between instrument variable and exposure is needed [20-22]. The genotype data of the 9 tested CORIN SNPs in 1472 individuals were used to calculate the LD correlation to construct the LD correlation matrix for GSMR analysis. The association threshold to select SNPs as the instruments in the GSMR analysis was set to be $5.56 \times 10^{-3}$ and the HEIDI-outlier threshold was 0.05 . For other parameters the default setting was used.

\section{Statistical analysis}

The differences of baseline characteristics between hypertensive cases and controls were compared, using Student's $t$-test for continuous variables and $\chi^{2}$ tests for categorical variables. The study participants were categorized into 4 groups according to the quartiles of serum corin levels. Odds ratios (ORs) and 95\% confidence intervals (CIs) of hypertension were calculated for upper quartiles of corin levels with the lowest quartile as references by using conditional logistic regression models. Potential covariates were smoking, drinking, BMI, TC, TG, HDL-C, FBG, and family history of hypertension. Hardy-Weinberg equilibrium (HWE) of the selected SNPs was tested with Fisher's exact test among control participants. Conditional logistic regression models were used to calculate ORs and $95 \%$ CIs of hypertension with SNPs. SNP was analyzed as 0,1 or 2 copies of the minor allele in an additive genetic model. Kruskal-Wallis test was applied to test the associations between the SNPs and baseline serum corin levels, and methylation levels. Statistical analyses were conducted using SAS statistical software version 9.2 (SAS Institute, Cary, NC).

\section{Results}

\section{Baseline characteristics}

In all, 731 hypertensive cases and 731 age- and sexmatched controls with full data of SNP genotypes, serum corin measurements were included in the analyses. Table 1 shows the baseline characteristics of the study cohort. There were $283(38.45 \%)$ men in each group. The mean age at baseline was 52.85 years old in hypertensive participants and 52.73 in controls. Compared with the controls, mean baseline BMI, SBP, DBP, TC, TG and FBG levels, follow-up SBP and DBP, and frequencies of drinkers were all higher in hypertensive patients $(P<0.05)$ (Table 1$)$. The mean SBP is 146.8 
Table 1 Characteristics of study participants

\begin{tabular}{|c|c|c|c|}
\hline Characteristics $^{\mathrm{a}}$ & Hypertensives $(n=731)$ & Controls $(n=731)$ & $P$ value \\
\hline Age, year & $52.85 \pm 12.79$ & $52.73 \pm 12.62$ & 0.8579 \\
\hline Male, \% & 38.45 & 38.45 & 1.000 \\
\hline Smokers, \% & 28.45 & 28.86 & 0.8622 \\
\hline Drinkers, \% & 23.94 & 19.02 & 0.0219 \\
\hline Family history of hypertension, $\%$ & 38.44 & 34.75 & 0.1427 \\
\hline Baseline SBP, mmHg & $126.4 \pm 8.37$ & $118.2 \pm 10.50$ & $<0.0001$ \\
\hline Baseline DBP, mmHg & $78.27 \pm 6.81$ & $72.93 \pm 7.25$ & $<0.0001$ \\
\hline Follow-up SBP, mmHg & $146.8 \pm 11.56$ & $121.1 \pm 10.54$ & $<0.0001$ \\
\hline Follow-up DBP, mmHg & $82.6 \pm 10.26$ & $70.67 \pm 7.99$ & $<0.0001$ \\
\hline $\mathrm{BMl}, \mathrm{kg} / \mathrm{m}^{2}$ & $22.89 \pm 3.27$ & $21.57 \pm 2.74$ & $<0.0001$ \\
\hline $\mathrm{TC}, \mathrm{mmol} / \mathrm{L}$ & $4.58 \pm 1.04$ & $4.47 \pm 0.91$ & 0.0366 \\
\hline $\mathrm{TG}, \mathrm{mmol} / \mathrm{L}$ & $1.58 \pm 1.13$ & $1.35 \pm 0.86$ & $<0.0001$ \\
\hline $\mathrm{HDL}-\mathrm{C}, \mathrm{mmol} / \mathrm{L}$ & $1.30 \pm 0.47$ & $1.34 \pm 0.48$ & 0.0972 \\
\hline LDL-C, mmol/L & $2.53 \pm 0.85$ & $2.46 \pm 0.74$ & 0.1245 \\
\hline $\mathrm{FBG}, \mathrm{mmol} / \mathrm{L}$ & $5.12 \pm 1.08$ & $5.01 \pm 0.81$ & 0.0306 \\
\hline Corin, pg/mL & $578.4 \pm 271.0$ & $585.4 \pm 258.5$ & 0.6157 \\
\hline
\end{tabular}

${ }^{a}$ : All variables presented are collected at baseline, except follow-up blood pressure

$\mathrm{mmHg}$ and mean DBP is $82.6 \mathrm{mmHg}$ in cases. It may be due to two reasons: 1) 62 individuals with $\mathrm{DBP} \geq 90$ $\mathrm{mmHg}$ but $\mathrm{SBP}<140 \mathrm{mmHg}$ and 437 individuals with $\mathrm{SBP} \geq 140 \mathrm{mmHg}$ but $\mathrm{DBP}<90 \mathrm{mmHg}$; 2) 239 patients took hypotensive drugs.

\section{Association between genotypes and corin levels and hypertension}

HWE of the 9 SNPs was tested with Fisher's exact test and no departure was observed (Table 2). Under additive models, we observed associations of rs2289433 (p.Cys13Tyr), rs6823184, rs10517195, rs2271037, and rs12509275 with serum corin levels after adjustment for covariates $(P=0.0399,0.0238,0.0016,0.0148$ and 0.0038 , respectively) (Table 2). However, if we considered multiple testing for the 9 CORIN SNPs, only the associations between rs10517195 and rs12509275 and serum corin levels were significant $(P<0.05 / 9=0.0056)$. Table 2 also showed the results of association between genotypes and hypertension. No significant association was detected in either univariate analysis or multivariate analysis.

Serum corin levels were lower in hypertensive patients $(578.4 \pm 271.0 \mathrm{pg} / \mathrm{mL})$ than in controls $(585.4 \pm 258.5 \mathrm{pg} /$ $\mathrm{mL}$ ) but not significant (Fig. 1). Conditional logistic regression models were used to assess the association of serum corin levels with hypertension. In univariate and

Table 2 Association between CORIN gene polymorphisms and serum corin levels and incident hypertension

\begin{tabular}{|c|c|c|c|c|c|c|c|c|c|c|c|c|}
\hline \multirow[t]{2}{*}{ SNP } & \multirow[t]{2}{*}{ Position } & \multirow[t]{2}{*}{ MA } & \multirow{2}{*}{$\begin{array}{l}\text { MAF } \\
\%\end{array}$} & \multirow[t]{2}{*}{$H W E^{*}$} & \multicolumn{4}{|l|}{ Corin } & \multicolumn{4}{|l|}{ Hypertension } \\
\hline & & & & & Beta & $P$ & Beta $^{\#}$ & $P^{\#}$ & OR(95\%Cl) & $P$ & $\mathrm{OR}(95 \% \mathrm{Cl})^{\#}$ & $P^{\#}$ \\
\hline rs2289433 & exon 1 & G & 34.3 & 0.075 & 0.035 & 0.052 & 0.037 & 0.040 & $1.08(0.92,1.26)$ & 0.358 & $1.13(0.96,1.34)$ & 0.152 \\
\hline rs6823184 & intron 1 & $C$ & 43.5 & 0.870 & 0.036 & 0.033 & 0.038 & 0.024 & $0.95(0.82,1.11)$ & 0.509 & $0.90(0.77,1.06)$ & 0.220 \\
\hline rs10008014 & intron 6 & $C$ & 24.6 & 0.289 & -0.002 & 0.883 & 0.000 & 0.990 & $0.99(0.84,1.18)$ & 0.931 & $1.02(0.85,1.22)$ & 0.853 \\
\hline rs17654423 & intron 6 & C & 26.6 & 0.670 & -0.020 & 0.510 & -0.019 & 0.319 & $1.02(0.87,1.20)$ & 0.801 & $1.02(0.86,1.22)$ & 0.804 \\
\hline rs10517195 & exon 9 & G & 15.7 & 0.167 & 0.074 & 0.001 & 0.074 & 0.002 & $0.94(0.77,1.16)$ & 0.568 & $0.93(0.76,1.16)$ & 0.515 \\
\hline rs2271037 & intron 9 & T & 38.7 & 0.551 & 0.042 & 0.022 & 0.042 & 0.015 & $1.01(0.87,1.17)$ & 0.911 & $0.97(0.83,1.14)$ & 0.736 \\
\hline rs2351784 & intron 11 & T & 23.0 & 0.335 & -0.014 & 0.615 & -0.012 & 0.854 & $0.92(0.77,1.10)$ & 0.348 & $0.90(0.75,1.09)$ & 0.288 \\
\hline rs12509275 & intron 17 & G & 13.8 & 0.064 & 0.073 & 0.003 & 0.070 & 0.004 & $1.01(0.82,1.25)$ & 0.913 & $0.94(0.75,1.18)$ & 0.573 \\
\hline rs3749585 & exon 22 & C & 48.0 & 0.774 & -0.001 & 0.814 & -0.006 & 0.737 & $0.99(0.85,1.14)$ & 0.852 & $0.98(0.84,1.15)$ & 0.834 \\
\hline
\end{tabular}

HWE Hardy-Weinberg equilibrium; MA Minor allele; MAF Minor allele frequency

*: $P$ value for HWE using Fisher's exact test

\#: Adjusted for smoking, drinking, BMI, TC, TG, HDL-C, FBG, family history of hypertension 


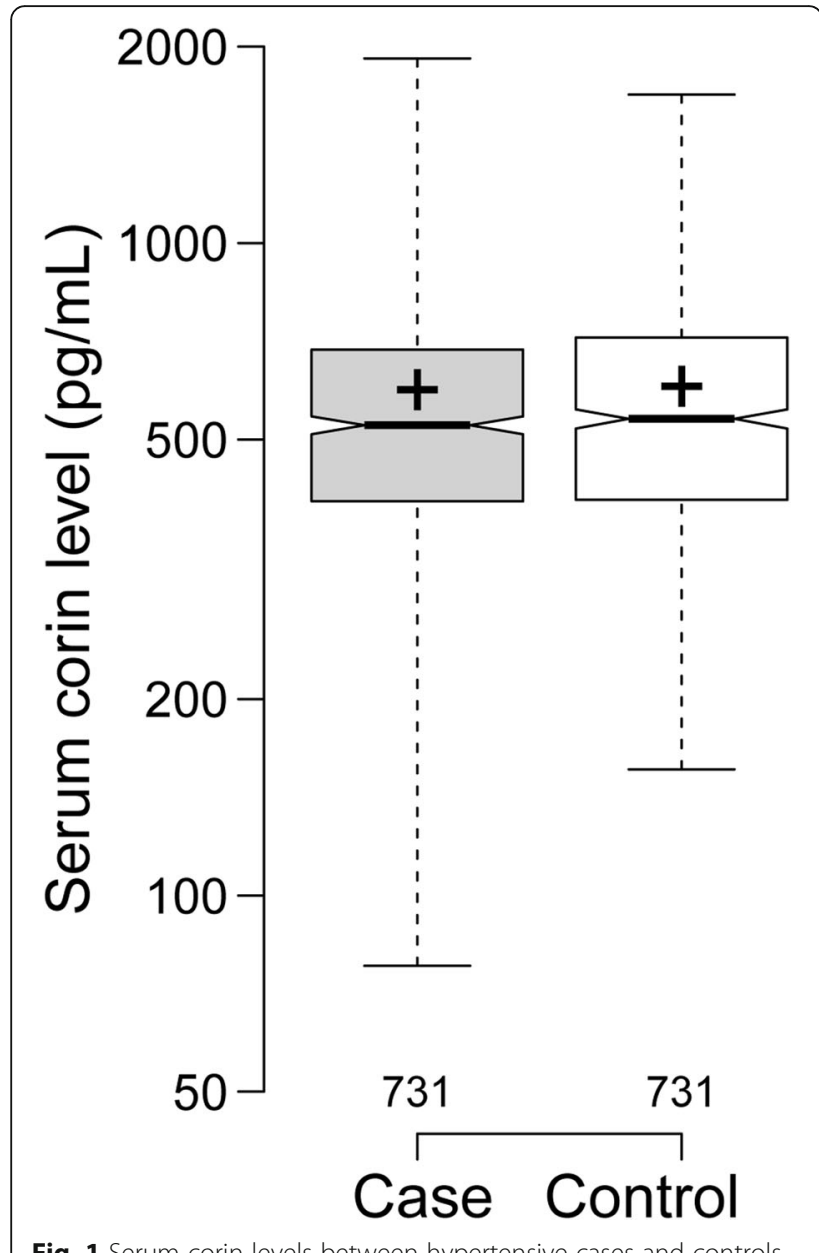

Fig. 1 Serum corin levels between hypertensive cases and controls

multivariate analysis, the association between serum corin levels and hypertension was not significant (Additional file 1: Table S1).

\section{Detection of functional variants}

The genetic association tests found 5 tag-SNPs that were associated with serum corin level. Therefore, we searched for functional variants which are in strong LD with the 5 corin-associated tag-SNPs, i.e., rs2289433, rs6823184, rs10517195, rs2271037 and rs12509275. There were 132 SNPs that are in strong LD with the 5 associated SNPs. So we focused on these 137 SNPs in the bioinformatics analysis (Additional file 2: Table S2). In HaploReg, we found that most of the SNPs overlap promoter, enhancer and DNase in multiple tissues. Several SNPs, such as tag-SNPs rs6823184, rs3215139, rs1344122, rs4695263 and rs4695264 might alter the binding of one or more proteins, and 126 SNPs might alter regulatory motifs in different cell types from the ENCODE transcription factor ChIP-seq datasets [23] (Additional file 2: Table S2). Among these SNPs, rs10049713, rs6823698, rs1866689 and the tag-SNPs rs6823184 were associated with expression of CORIN gene in whole blood cells (GTEx 2015_v6) (the top $P$ values $=2.38 \times 10^{-24}, \quad 5.94 \times 10^{-27}, \quad 6.31 \times 10^{-27}$ and $6.30 \times 10^{-27}$, respectively). The associations in other tissue such as Esophagus Muscularis were also observed. These four SNPs locate in intron 1 of CORIN. We found that rs6823698 locate in a DNase hypersensitivity cluster, and rs6823184 locate in a DNase hypersensitivity cluster, a $\mathrm{CpG}$ island and transcription factor binding sites (Fig. 2).

\section{Causal inference}

According to the bioinformatics analysis, we noticed that the corin-associated SNPs locate around a CpG island. So we tested the associations between CORIN SNPs and methylation levels. Twenty-five methylation sites in CORIN were analyzed. We found 12 significant (FDR < 0.05 ) associations between 12 SNPs and cg02955940 methylation sites in CORIN gene (Fig. 2, Additional file 3: Table S3). The associations between the 9 tag-SNPs genotyped in our study and methylation levels were evaluated. Four of the CORIN tag-SNPs seemed to be associated with cg02955940 $\left(P<2.54 \times 10^{-3}\right)$. For the corinassociated tag-SNPs, the association between rs6823184 and cg02955940 methylation level was the most significant $\left(P=1.54 \times 10^{-7}\right)$ (Fig. 3a, Additional file 4: Table S4). We used these 9 CORIN SNPs that have data on both of the associations of SNPs with serum corin levels and the associations of SNPs with methylation levels as genetic instruments in Mendelian randomization analysis. Four SNPs that were significantly associated with methylation $\left(P<2.54 \times 10^{-3}\right)$ were retained after filtering and passed the HEIDI-outlier test. Thus, these four SNPs were the instrumental variables in the GSMR analysis. The estimated effect of cg02955940 methylation on corin levels was 0.035 (standard error $=0.0108, P=0.0011$ ) (Fig. 3b). This result suggested a putative causal association between cg02955940 methylation and corin levels.

\section{Discussion}

This is the first study to investigate the relationships among CORIN SNPs and methylations, serum corin levels and risk of hypertension in China. Our study suggested that CORIN SNPs were associated with serum corin levels and cg02955940 methylation was likely to be a causal factor for corin levels.

Human ANP exerts an important role in regulation of systemic BP and intravascular volume by enhancing vascular permeability $[24,25]$. Low ANP level is a risk factor for hypertension $[26,27]$. Deficiency in corin leads to reduced ANP production [5]. The importance of corin in regulating $\mathrm{BP}$ has been evaluated. In corin-null mice, SBP, DBP and mean arterial BP were increased compared to the normal controls [5]. Corin has been 


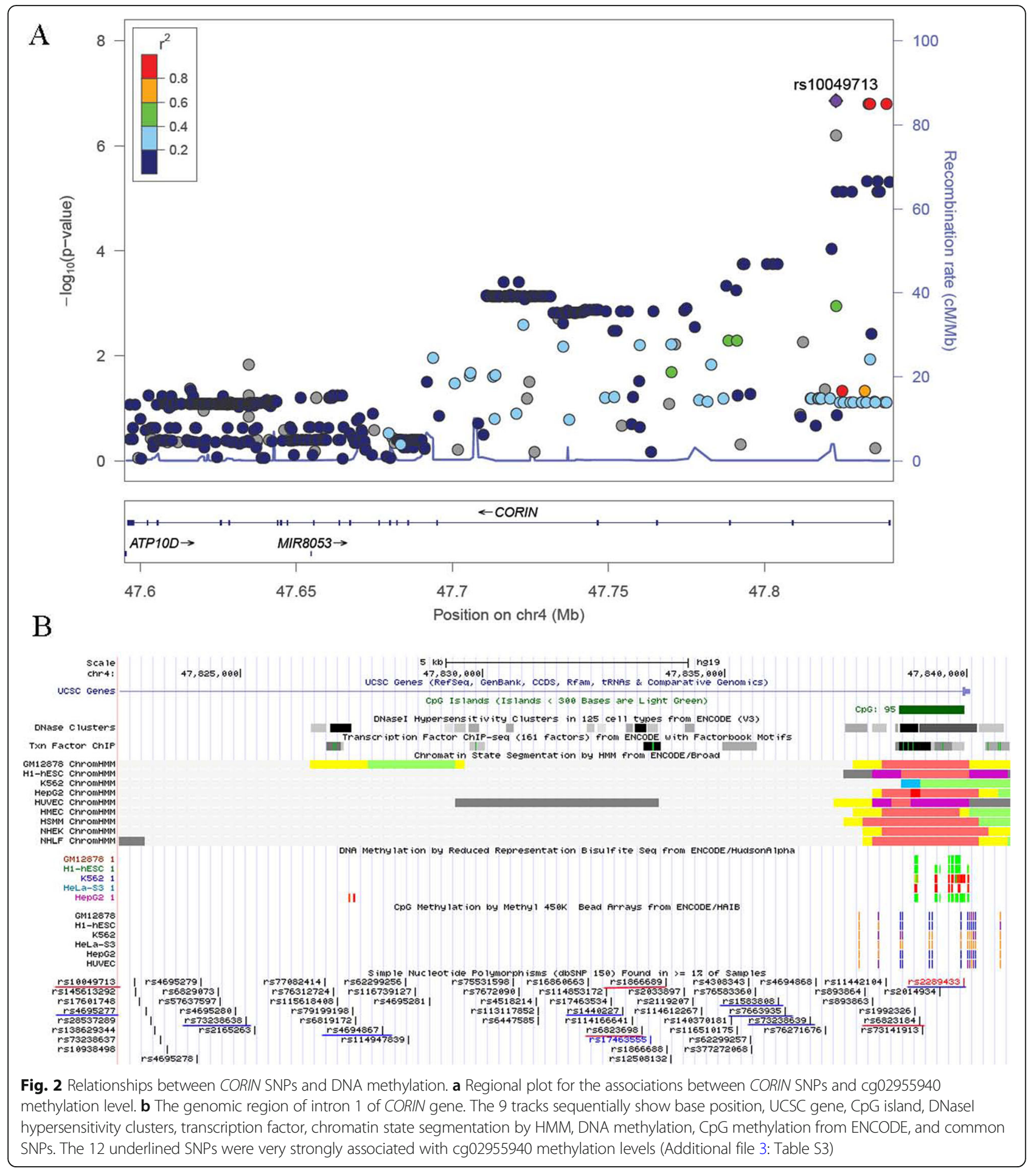

reported to be a valuable prognostic marker of major adverse cardiac events in patients with acute myocardial infarction, independent of established conventional risk factors [28]. A previous cross-sectional study has detected the significant higher level of serum soluble corin in hypertensive patients [7], which was inconsistent with the hypothesis from cell- and animal-based studies that increased corin level probably lowered BP $[5,6]$. In the present study, we prospectively evaluated the association between baseline corin levels and incident hypertension but did not detect a significant association. However, the mean corin level of hypertension patients at baseline was lower than that of normotensive participants, which was consistent with the hypothesis. Corin is a converting 

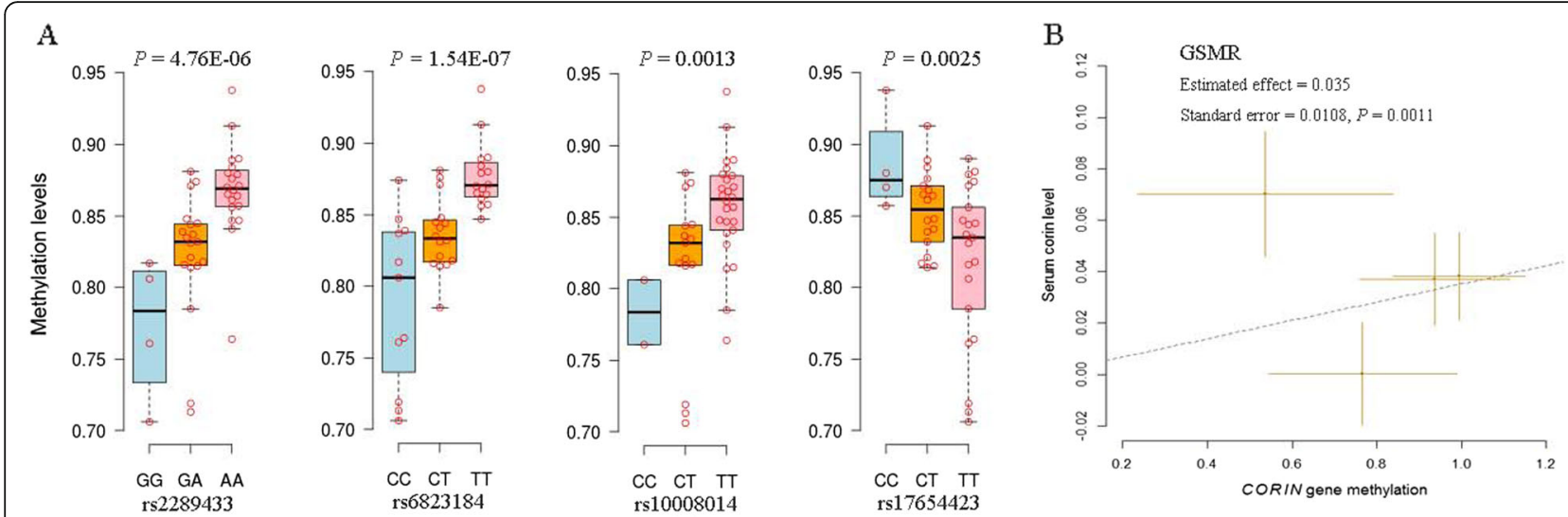

Fig. 3 Relationship between CORIN SNPs, methylation and corin levels. a Four SNPs, including rs2289433, rs6823184, rs10008014 and rs17654423, were significantly associated with cg02955940 methylation levels. b We detected a putative causal association between cg02955940 methylation and corin levels by using these CORIN SNPs as genetic instruments in Mendelian randomization analysis (Additional file 4: Table S4)

enzyme in the natriuretic peptide system. Changes in activity or concentration of this convertase would affect ANP concentration and then influence hypertension risk. Even though, the function of this protein on BP regulation should not be as direct as ANP. The effect size of corin on hypertension incidence may be too small to detect.

The associations between CORIN gene SNPs and serum corin levels have not been comprehensively assessed in previous studies. The two well-known polymorphisms, T555I and Q568P, are most likely to be monomorphisms in the Chinese Han population (we have also genotyped these two SNPs in 1480 individuals). The effects of these two variants can't be evaluated in our study. Taking no account of these two SNPs, we identified several SNPs that were significantly associated with serum corin level. Moreover, the effects of these SNPs on corin level may be mediated by DNA methylation. Therefore, our data suggested that genetic variants in CORIN gene may affect serum corin levels. To our knowledge, these associations were first reported.

In African Americans, Dries et al. had reported the associations of CORIN variants (T555I and Q568P) with hypertension and heart disease [29]. Later, other polymorphisms have been reported [30]. But these associations have not been confirmed in recent large scale genome-wide association studies. A recent study evaluated the associations of three CORIN SNPs and BP in a Chinese population but no significant association was detected [9], while another study concluded that rs2271037 and rs3749585 were significantly associated with hypertension in a Han population of northeastern China [8]. Although data from several studies suggested that CORIN variants may contribute to hypertension and heart disease in this high-risk population [29, 31-33], the association between CORIN gene variants and incident hypertension has not yet been reported. We evaluated the association between CORIN gene variants and incident hypertension but fail to detect a significant result. Given the fact that BP is a complex trait, which influenced by multiple interacting physiologic regulatory systems where multiple genes are likely to be involved, small genetic effects can be expected. The effects of CORIN gene variants on hypertension risk were indirect and would be very small.

This study has several strong points. First, we investigated the association between baseline serum corin level and the risk of incident hypertension in a nested casecontrol study. The prospective design reduced the potential biases inherent in cross-sectional or retrospective studies. Second, simultaneously testing several tag-SNPs and serum corin levels in a sample ensured us a comprehensive analysis of the relationships among SNPs, corin levels and hypertension incidence. Finally, and most importantly, we found potentially functional variants in the CORIN gene according to data from functional annotation databases and data from large projects such as ENCODE, which might consequently facilitate the interpretation of the associations. The observation that corin-associated SNPs locate in a CpG island guided us to evaluate the relationship among SNPs, methylations and corin levels, which enables us to discover the causal effect of methylation on corin level.

Although we were not able to link serum corin levels to hypertension in a Chinese Han population, potentially functional SNPs were found to be significantly associated to serum corin level, and the effect of these SNPs on corin level may be mediated by DNA methylation. These findings were first reported. Previous findings in cells, animals and the general populations supported the interest of investigations on corin, but the regulatory mechanism is far from fully understood. Our findings provided new clues for 
further investigation in order to unfold the mechanisms involved.

Our study has some potential limitations. First, the study sample was too small to detect the associations between CORIN SNPs and hypertension. We displayed power estimations by using the CaTS software. We found that no SNP has enough power $(<80 \%)$ to detect the association in an additive model. Larger samples are needed to detect the association between CORIN SNPs and hypertension. For example, we have $38 \%$ power to detect the association between rs16823124 and hypertension in the current samples. For this SNP, if the sample size is increased to thousands of individuals (3000 cases and 3000 controls) the statistical power may be high enough (84\%). Second, we did not test the corin activities or gene expressions to evaluate the effects. However, several molecular genetic studies have shown that the CORIN mutations may adversely affect the biological activity of corin [30,31,33], and we have detected ciseQTL effects for these SNPs. Third, although we detected the strong associations between CORIN SNPs and methylation, the sample is relatively small. The relationship among SNPs, methylations and corin levels needs to be confirmed in larger samples.

\section{Conclusion}

In summary, this is the first study to show that potentially functional variants in CORIN gene were associated with serum corin level in the Chinese Han population. The effect of these SNPs on corin level may be mediated by CORIN methylation. Further studies are needed to confirm the associations and elucidate the mechanism.

\section{Supplementary information}

Supplementary information accompanies this paper at https://doi.org/10. 1186/s12863-019-0802-4.

Additional file 1: Table S1. Odds ratios and 95\% confidence interval of hypertension associated with quartiles of Corin. The results showed that in univariate and multivariate analysis, the association between serum corin levels and hypertension was not significant.

Additional file 2: Table S2. Genetic variants in strong linkage disequilibrium with the corin-associated SNPs. There were 132 SNPs that are in strong LD with the 5 corin-associated SNPs. This table showed the bioinformatics analysis results for these 137 SNPs.

Additional file 3: Table S3. Significant associations between CORIN SNPs and methylations. The results showed 12 significant associations between 12 SNPs and cg02955940 methylation sites in CORIN gene $($ FDR $<0.05)$

Additional file 4: Table S4. Summary association data for GSMR analysis. This table contained the formatted summary-level data that was used in the GSMR analysis.

\section{Abbreviations}

ANP: A-type natriuretic peptides; BMI: Body mass index; BP: Blood pressure; $\mathrm{Cl}$ : Confidence intervals; DBP: Diastolic blood pressure; eQTL: Expression quantitative trait loci; FBG: Fasting blood glucose; GSMR: Generalised Summary-data-based Mendelian Randomization; HDL-C: High-density lipoprotein cholesterol; LD: Linkage disequilibrium; MAF: Minor allele frequency; OR: Odds ratios; SBP: Systolic blood pressure; SNP: Single nucleotide polymorphism; TC: Total cholesterol; TG: Triglycerides

\section{Acknowledgements}

Not Applicable.

\section{Authors' contributions}

$H Z, X B M$ and $Y H Z$ had the original idea for the study. SFL and TX jointly participated in its design, interpreted the data and critically revised the article. QYQ, ZBZ, XFHF and ALW took part in the acquisition of data. HZ and XBM performed the statistical analysis and drafted the work. ZYZ and ZRG revised the manuscript critically for important intellectual content. YHZ provided supervision. All authors have approved the submitted version and have agreed both to be personally accountable for the author's own contributions and to ensure that questions related to the accuracy or integrity of any part of the work.

\section{Funding}

The work was supported by Natural Science Foundation of China $(81773508$ 81302499 and 81320108026), the Key Research Project (Social Development Plan) of Jiangsu Province (BE2016667), Project funded by China Postdoctoral Science Foundation (2014 T70547, 2013 M530269 and 2014 M551649), the Startup Fund from Soochow University (Q413900313, Q413900412), and a Project of the Priority Academic Program Development of Jiangsu Higher Education Institutions.

\section{Availability of data and materials}

The datasets supporting the conclusions of this article are included within the article and its additional files.

Ethics approval and consent to participate

This study was approved by the ethics committee at Soochow University in China. Written informed consent was obtained from each of the study participants.

\section{Consent for publication}

Not applicable

\section{Competing interests}

The authors declare that they have no competing interests.

\section{Author details}

${ }^{1}$ Jiangsu Key Laboratory of Preventive and Translational Medicine for Geriatric Diseases, Medical College of Soochow University, Suzhou, Jiangsu, People's Republic of China. ${ }^{2}$ Department of Epidemiology, School of Public Health, Medical College of Soochow University, Suzhou, 199 Renai Road, Industrial Park District, Suzhou 215123, Jiangsu, People's Republic of China. ${ }^{3}$ Center for Genetic Epidemiology and Genomics, School of Public Health, Medical College of Soochow University, Suzhou, Jiangsu, People's Republic of China. ${ }^{4}$ Changshu Center of Disease Control and Prevention, Suzhou, Jiangsu, People's Republic of China.

Received: 5 November 2018 Accepted: 15 December 2019

Published online: 19 December 2019

\section{References}

1. Poulter NR, Prabhakaran D, Caulfield M. Hypertension. Lancet. 2015; 386(9995):801-12.

2. Kearney PM, Whelton M, Reynolds K, Muntner P, Whelton PK, He J. Global burden of hypertension: analysis of worldwide data. Lancet. 2005;365(9455): 217-23.

3. Lewington S, Lacey B, Clarke R, Guo Y, Kong XL, Yang L, et al. The burden of hypertension and associated risk for cardiovascular mortality in China. JAMA Intern Med. 2016:176(4):524-32.

4. Levin ER, Gardner DG, Samson WK. Natriuretic peptides. N Engl J Med. 1998; 339(5):321-8

5. Chan JC, Knudson O, Wu F, Morser J, Dole WP, Wu Q. Hypertension in mice lacking the proatrial natriuretic peptide convertase corin. Proc Natl Acad Sci U S A. 2005;102(3):785-90. 
6. Zhou Y, Wu Q. Corin in natriuretic peptide processing and hypertension. Curr Hypertens Rep. 2014;16(2):415.

7. Peng H, Zhang Q, Cai X, Liu Y, Ding J, Tian H, et al. Association between high serum soluble Corin and Hypertension: a cross-sectional study in a general population of China. Am J Hypertens. 2015;28(9):1141-9.

8. Chen YL, Li TJ, Hao Y, Wu BG, Li H, Geng N, et al. Association of rs2271037 and rs3749585 polymorphisms in CORIN with susceptibility to hypertension in a Chinese Han population: a case-control study. Gene. 2018;651:79-85.

9. Hu BC, Li Y, Liu M, Li LH, Sheng CS, Zhang Y, et al. Blood pressure and urinary sodium excretion in relation to 16 genetic polymorphisms in the natriuretic peptide system in Chinese. Endocr J. 2014;61(9):861-74.

10. Zhang $Y$, Zhou T, Niu Y, He M, Wang C, Liu M, et al. Identification and functional analysis of CORIN variants in hypertensive patients. Hum Mutat. 2017:38(12):1700-10.

11. Xu T, Zhong C, Wang A, Guo Z, Bu X, Zhou Y, et al. YKL-40 level and hypertension incidence: a population-based nested case-control study in China. J Am Heart Assoc. 2016;5(11):e004534.

12. Zhang H, Mo X, Zhou Z, Zhu Z, HuangFu X, Xu T, et al. Associations among NPPA gene polymorphisms, serum ANP levels, and hypertension in the Chinese Han population. J Hum Hypertens. 2019;33(9):641-7.

13. James PA, Oparil S, Carter BL, Cushman WC, Dennison-Himmelfarb C, Handler J, et al. 2014 evidence-based guideline for the management of high blood pressure in adults: report from the panel members appointed to the eighth joint National Committee (JNC 8). JAMA. 2014; 311(5):507-20.

14. Barrett JC, Fry B, Maller J, Daly MJ. Haploview: analysis and visualization of LD and haplotype maps. Bioinformatics. 2005;21(2):263-5.

15. Zhu Z, Zheng Z, Zhang F, Wu Y, Trzaskowski M, Maier R, et al. Causal associations between risk factors and common diseases inferred from GWAS summary data. Nat Commun. 2018;9(1):224.

16. Ren J, Jiang C, Gao X, Liu Z, Yuan Z, Jin C, et al. PhosSNP for systematic analysis of genetic polymorphisms that influence protein phosphorylation. Mol Cell Proteomics. 2010;9(4):623-34.

17. Mo XB, Wu LF, Zhu XW, Xia W, Wang L, He P, et al. Identification and evaluation of IncRNA and mRNA integrative modules in human peripheral blood mononuclear cells. Epigenomics. 2017;9(7):943-54.

18. Shabalin AA. Matrix eQTL: ultra fast eQTL analysis via large matrix operations. Bioinformatics. 2012;28(10):1353-8.

19. Zhu Z, Zhang F, Hu H, Bakshi A, Robinson MR, Powell JE, et al. Integration of summary data from GWAS and eQTL studies predicts complex trait gene targets. Nat Genet. 2016;48(5):481-7.

20. Hannon E, Gorrie-Stone TJ, Smart MC, Burrage J, Hughes A, Bao Y, et al. Leveraging DNA-methylation quantitative-trait loci to characterize the relationship between Methylomic variation, gene expression, and complex traits. Am J Hum Genet. 2018;103(5):654-65.

21. Xue A, Wu Y, Zhu Z, Zhang F, Kemper KE, Zheng Z, et al. Genome-wide association analyses identify 143 risk variants and putative regulatory mechanisms for type 2 diabetes. Nat Commun. 2018;9(1):2941.

22. Qi T, Wu Y, Zeng J, Zhang F, Xue A, Jiang L, et al. Identifying gene targets for brain-related traits using transcriptomic and methylomic data from blood. Nat Commun. 2018:9(1):2282

23. Kheradpour P, Kellis M. Systematic discovery and characterization of regulatory motifs in ENCODE TF binding experiments. Nucleic Acids Res. 2014;42(5):2976-87.

24. Potter LR, Yoder AR, Flora DR, Antos LK, Dickey DM. Natriuretic peptides: their structures, receptors, physiologic functions and therapeutic applications. Handb Exp Pharmacol. 2009;191:341-66.

25. Curry FR. Atrial natriuretic peptide: an essential physiological regulator of transvascular fluid, protein transport, and plasma volume. J Clin Invest. 2005; 115(6):1458-61.

26. Conen D, Glynn RJ, Buring JE, Ridker PM, Zee RY. Natriuretic peptide precursor a gene polymorphisms and risk of blood pressure progression and incident hypertension. Hypertens. 2007;50(6):1114-9.

27. John SW, Krege JH, Oliver PM, Hagaman JR, Hodgin JB, Pang SC, et al. Genetic decreases in atrial natriuretic peptide and salt-sensitive hypertension. Sci. 1995;267(5198):679-81.

28. Zhou X, Chen J, Zhang Q, Shao J, Du K, Xu X, et al. Prognostic value of plasma soluble Corin in patients with acute myocardial infarction. J Am Coll Cardiol. 2016;67(17):2008-14.

29. Dries DL, Victor RG, Rame JE, Cooper RS, Wu X, Zhu X, et al. Corin gene minor allele defined by 2 missense mutations is common in blacks and associated with high blood pressure and hypertension. Circ. 2005;112(16): 2403-10.

30. Dong N, Fang C, Jiang Y, Zhou T, Liu M, Zhou J, et al. Corin mutation R539C from hypertensive patients impairs zymogen activation and generates an inactive alternative ectodomain fragment. J Biol Chem. 2013;288(11):7867-74.

31. Rame JE, Drazner MH, Post W, Peshock R, Lima J, Cooper RS, et al. Corin I555(P568) allele is associated with enhanced cardiac hypertrophic response to increased systemic afterload. Hypertens. 2007;49(4):857-64.

32. Rame JE, Tam SW, McNamara D, Worcel M, Sabolinski ML, Wu AH, et al. Dysfunctional corin i555(p568) allele is associated with impaired brain natriuretic peptide processing and adverse outcomes in blacks with systolic heart failure: results from the genetic risk assessment in heart failure substudy. Circ Heart Fail. 2009;2(6):541-8.

33. Wang W, Liao X, Fukuda K, Knappe S, Wu F, Dries DL, et al. Corin variant associated with hypertension and cardiac hypertrophy exhibits impaired zymogen activation and natriuretic peptide processing activity. Circ Res. 2008;103(5):502-8.

\section{Publisher's Note}

Springer Nature remains neutral with regard to jurisdictional claims in published maps and institutional affiliations.

\section{Ready to submit your research? Choose BMC and benefit from:}

- fast, convenient online submission

- thorough peer review by experienced researchers in your field

- rapid publication on acceptance

- support for research data, including large and complex data types

- gold Open Access which fosters wider collaboration and increased citations

- maximum visibility for your research: over $100 \mathrm{M}$ website views per year

At BMC, research is always in progress.

Learn more biomedcentral.com/submissions 